\title{
Performance Evaluation of Cognitive Multi-Relay Networks with Multi-Receiver Scheduling
}

\author{
Thi My Chinh Chu ${ }^{\dagger}$, Hans-Jürgen Zepernick ${ }^{\dagger}$, and Hoc Phan* \\ ${ }^{\dagger}$ Blekinge Institute of Technology, Karlskrona, Sweden, E-mail: \{thi.my.chinh.chu, hans-jurgen.zepernick\}@bth.se \\ ${ }^{*}$ University of Reading, RG6 6AY, UK, E-mail: h.phan@ reading.ac.uk
}

\begin{abstract}
In this paper, we investigate the performance of cognitive multiple decode-and-forward relay networks under the interference power constraint of the primary receiver wherein the cognitive downlink channel is shared among multiple secondary relays and secondary receivers. In particular, only one relay and one secondary receiver which offers the highest instantaneous signal-to-noise ratio is scheduled to transmit signals. Accordingly, only one transmission route that offers the best end-to-end quality is selected for communication at a particular time instant. To quantify the system performance, we derive expressions for outage probability and symbol error rate over Nakagami- $m$ fading with integer values of fading severity parameter $m$. Finally, numerical examples are provided to illustrate the effect of system parameters such as fading conditions, the number of secondary relays and secondary receivers on the secondary system performance.
\end{abstract}

\section{INTRODUCTION}

Nowadays, the increasing demand on high data rate services leads to exhausted frequency resources. Despite this shortage, radio spectrum is still under-utilized [1] which has fostered studies to improve spectrum efficiency. Specifically, cognitive radio $(\mathrm{CR})$ has emerged as a promising approach to improve spectrum utilization [2]-[5]. The studies of [2], [3] discussed crucial requirements of CRs such as spectrum hole detection, channel state estimation, interference temperature estimation, transmit power control, and dynamic spectrum access. In general, there are three spectrum access strategies for cognitive radio networks (CRNs), i.e., the overlay, underlay, and interweave schemes. Techniques for adjusting secondary transmit powers to meet the interference power constraint of the primary receiver in underlay CRNs are presented in [5] while collaborative spectrum sensing techniques for interweave CRNs are studied in [6]. Dealing with the overlay scheme, the authors of [7] proposed a power allocation for CRNs to achieve maximum rate whereas a hybrid scheme was deployed in [8] to inherit the benefits of the interweave and underlay schemes.

Cooperative communications has been recognized as a powerful technique to provide transmission reliability and to extend radio coverage. This technique deploys one or multiple relay nodes between the transmitter and receiver to forward the source signals to the destination. In traditional relay communication, the system is concerned with pointto-point links each of which has a single source and a single destination [9]. However, in environments with severe shadowing between the transmitter and receiver, point-to-point communication has difficulties to provide high data rates for the secondary transmission. This typically occurs in underlay CRNs where the transmit powers are strictly controlled by the interference power constraints imposed by primary networks. In this case, cooperative communications can be used for the cognitive downlink to improve the quality of service of CRNs. The works of [10], [11] proposed relaying policies to select a suitable relay among relay candidates based on the received signal-to-noise ratio (SNR) of an underlay cognitive cooperative radio network (CCRN). However, all the works of [10], [11] performed a transmission from a single secondary transmitter to a signal secondary receiver.

Hence, the objective of this paper is to investigate the performance of a CRN which jointly includes multiple relaying and scheduling transmission for multiple secondary receivers. In this network, only one relay and one secondary receiver which offer the highest instantaneous SNR is scheduled for transmission. We utilize the decode-and-forward (DF) relaying scheme since it is able to cancel the noise at the relays. This advantage is crucial in underlay cognitive radio networks where transmit powers of the secondary network are often kept rather low to satisfy the interference power constraint of the primary receiver [5]. To quantify the system performance, we derive expressions for outage probability and symbol error rate (SER) in case of Nakagami- $m$ fading with integer fading severity parameter $m$. Based on our analysis, the impact of the fading conditions, the number of secondary relays and secondary receivers on outage probability and SER is revealed.

Notation: This paper uses the following notations. The probability density function (PDF) and the cumulative distribution function (CDF) of a random variable (RV) $X$ are denoted as $f_{X}(\cdot)$ and $F_{X}(\cdot)$, respectively. The gamma function [12, eq. (8.310.1)] is presented by $\Gamma(n)$. Furthermore, $U(a, b ; x)$ is the confluent hypergeometric function [12, eq. (9.211.4)] and $E\{\cdot\}$ stands for the expectation operator. Finally, $C_{k}^{n}=$ $\frac{n !}{k !(n-k) !}$ is the binomial coefficient.

\section{System And Channel Model}

Consider the downlink of a cognitive CCRN with underlay spectrum access that is subject to the interference power constraint $Q$ of a primary receiver $\mathrm{PU}_{\mathrm{RX}}$ over independent and identically distributed (i.i.d.) Nakagami- $m$ fading channels. The CCRN consists of a secondary transmitter $\mathrm{SU}_{\mathrm{TX}}$, which is scheduled to transmit signals to $K$ secondary receivers, $\mathrm{SU}_{\mathrm{RX}, 1}, \ldots, \mathrm{SU}_{\mathrm{RX}, K}$, through the support of $L \mathrm{DF}$ relays, 


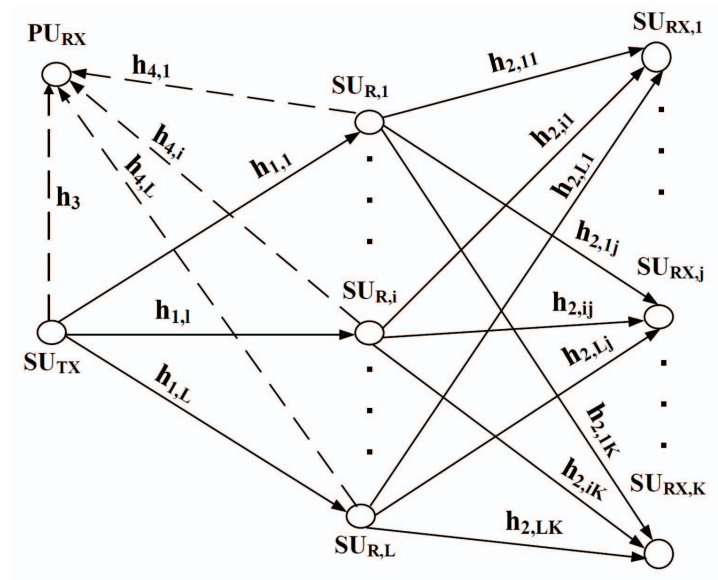

Fig. 1. System model of a cognitive multi-relay network with multi-receiver scheduling.

$\mathrm{SU}_{\mathrm{R}, 1}, \ldots, \mathrm{SU}_{\mathrm{R}, L}$, as depicted in Fig. 1. In this figure, $h_{1, i}$, $i=1, \ldots, L$, is the channel coefficient of the link from $\mathrm{SU}_{\mathrm{TX}}$ to $\mathrm{SU}_{\mathrm{R}, i}$. Furthermore, $h_{2, l k}$ is the channel coefficient of the link from $\mathrm{SU}_{\mathrm{R}, l}, l=1, \ldots, L$, to $\mathrm{SU}_{\mathrm{RX}, k}, k=1, \ldots, K$. Next, $h_{3}$ is the channel coefficient of the link $\mathrm{SU}_{\mathrm{TX}}$ to $\mathrm{PU}_{\mathrm{RX}}$. Finally, $h_{4, i}, i=1, \ldots, L$, is the channel coefficient of the link from $\mathrm{SU}_{\mathrm{R}, i}$ to $\mathrm{PU}_{\mathrm{RX}}$. Due to shadowing, we assume that the direct link between the secondary transmitter and receiver is absent.

In this system, time division multiple access (TDMA) is used to share the downlink channel among $K$ secondary receivers. Specifically, only one relay and one secondary receiver which offer the highest instantaneous signal-to-noise ratio (SNR) is scheduled for transmission. This means that the transmission route that has the most favorable end-toend quality is selected for communication. Furthermore, it is assumed that relaying transmission is performed in halfduplex mode, i.e., the communication period is divided into two consecutive time slots. In the first time slot, the secondary transmitter sends a signal to the selected relay. Let $x$ be the transmit signal at $\mathrm{SU}_{\mathrm{TX}}$. The average transmit power at the secondary transmitter must be controlled to satisfy the interference power constraint $Q$ of the primary receiver $\mathrm{PU}_{\mathrm{RX}}$, i.e., $P_{s}=E\left\{|x|^{2}\right\}=Q /\left|h_{3}\right|^{2}$. Then, the received signal at the $l^{\text {th }}$ relay, $\mathrm{SU}_{\mathrm{R}, l}$, is expressed as

$$
y_{R, l}=h_{1, l} x+n_{R, l}
$$

where $n_{R, l}$ is the additive white Gaussian noise (AWGN) at $\mathrm{SU}_{\mathrm{R}, l}$ with zero mean and variance $N_{0}$. As a result, the instantaneous SNR at the $l^{\text {th }}$ relay, $\mathrm{SU}_{\mathrm{R}, l}$, is obtained as

$$
\gamma_{1, l}=\beta X_{1, l} / X_{3}
$$

where $\beta$ is determined as $\beta=Q / N_{0}$. Further, $X_{1, l}=\left|h_{1, l}\right|^{2}$ and $X_{3}=\left|h_{3}\right|^{2}$ are the channel power gains of the links $\mathrm{SU}_{\mathrm{TX}} \rightarrow \mathrm{SU}_{\mathrm{R}, l}$ and $\mathrm{SU}_{\mathrm{TX}} \rightarrow \mathrm{PU}_{\mathrm{RX}}$, respectively.

In the second time slot, the relay decodes the received signal and sends the resulting signal $x_{l}$ to the $k^{\text {th }}$ selected secondary receiver, $\mathrm{SU}_{\mathrm{RX}, k}$. The average transmit power at $\mathrm{SU}_{\mathrm{R}, l}$ must be regulated as $P_{l}=E\left\{\left|x_{l}\right|^{2}\right\}=Q /\left|h_{4, l}\right|^{2}$. Then, the received signal at $\mathrm{SU}_{\mathrm{RX}, k}$ from $\mathrm{SU}_{\mathrm{R}, l}$ is given by

$$
y_{D, l k}=h_{2, l k} x_{l}+n_{D, k}
$$

where $n_{D, k}$ is the AWGN at $\mathrm{SU}_{\mathrm{RX}, k}$ with zero mean and variance $N_{0}$. Thus, the instantaneous SNR at the $k^{t h}$ secondary receiver, $\mathrm{SU}_{\mathrm{RX}, k}$, is obtained as

$$
\gamma_{2, l k}=\beta X_{2, l k} / X_{4, l}
$$

where $X_{2, l k}=\left|h_{2, l k}\right|^{2}$ and $X_{4, l}=\left|h_{4, l}\right|^{2}$ are the channel power gains of the links $\mathrm{SU}_{\mathrm{R}, l} \rightarrow \mathrm{SU}_{\mathrm{RX}, k}$ and $\mathrm{SU}_{\mathrm{R}, l} \rightarrow \mathrm{PU}_{\mathrm{RX}}$, respectively.

Among the $K$ secondary receivers, the destination which obtains the highest instantaneous SNR from the respective selected relay is selected for communication, i.e., the instantaneous SNR from the selected relay to the $k^{\text {th }}$ selected secondary receiver is obtained as

$$
\gamma_{2, l}=\max _{1 \leq k \leq K}\left(\gamma_{2, l k}\right)
$$

Since DF is utilized at $\mathrm{SU}_{\mathrm{R}, l}$, the end-to-end $\mathrm{SNR}$ at the $k^{t h}$ secondary receiver is found as $\min \left(\gamma_{1, l}, \gamma_{2, l}\right)$ [13]. Furthermore, the relay $\mathrm{SU}_{\mathrm{R}, l}$ will be selected for communication only if the transmission through this relay offers the highest SNR among all the possible routes. Thus, the end-to-end SNR from $\mathrm{SU}_{\mathrm{TX}}$ to the selected secondary receiver is obtained as

$$
\gamma_{D}=\max _{1 \leq l \leq L}\left\{\min \left[\gamma_{1, l}, \max _{1 \leq k \leq K}\left(\gamma_{2, l k}\right)\right]\right\}
$$

As such, we can rewrite $\gamma_{D}$ as

$$
\gamma_{D}=\max _{1 \leq l \leq L}\left[\min \left(\gamma_{1, l}, \gamma_{2, l}\right)\right]=\max _{1 \leq l \leq L}\left(\gamma_{l}\right)
$$

where $\gamma_{l}$ is defined as

$$
\gamma_{l}=\min \left[\gamma_{1, l}, \max _{1 \leq l \leq K}\left(\gamma_{2, l k}\right)\right]
$$

Before further analyzing the system performance, we need to provide the CDF and PDF of the channel power gain of a Nagakami- $m$ fading channel with integer fading severity $m_{i}$ and channel mean power $\Omega_{i}$ as

$$
\begin{aligned}
& F_{X_{i}}(x)=1-\exp \left(-\alpha_{i} x\right) \sum_{i=0}^{m_{i}-1} \frac{\alpha_{i}^{i} x^{i}}{i !} \\
& f_{X_{i}}(x)=\frac{\alpha_{i}^{m_{i}}}{\Gamma\left(m_{i}\right)} x^{m_{i}-1} \exp \left(-\alpha_{i} x\right)
\end{aligned}
$$

where $\alpha_{i}=m_{i} / \Omega_{i}$.

\section{END-To-End PeRformance AnALysis}

In order to obtain the outage probability and SER of the system, we must derive an expression for the CDF of the instantaneous SNR $\gamma_{D}$ of the system. As can be seen from (7), $\gamma_{D}$ is expressed as a function of $\gamma_{1, l}, l \in\{1, \ldots, L\}$. From (2), all $\gamma_{1, l}$ contain the same variable $X_{3}$ which leads to statistical dependence among $\gamma_{1, l}$ with $l \in\{1, \ldots, L\}$. Thus, we first calculate $F_{\gamma_{D}}\left(\left.\gamma\right|_{X_{3}}\right)$, then, we obtain $F_{\gamma_{D}}(\gamma)$ as the expectation of $F_{\gamma_{D}}\left(\left.\gamma\right|_{X_{3}}\right)$ over the PDF of $X_{3}$. Based on the order statistics theory, the CDF of $\gamma_{D}$ conditioned on $X_{3}$ can 
be found from (7) as

$$
F_{\gamma_{D}}\left(\gamma \mid X_{3}\right)=\left[F_{\gamma_{l}}\left(\gamma \mid X_{3}\right)\right]^{L}
$$

From (8), $F_{\gamma_{l}}\left(\gamma \mid X_{3}\right)$ can be calculated as

$$
F_{\gamma_{l}}\left(\gamma \mid X_{3}\right)=1-\left[1-F_{\gamma_{1, l}}\left(\gamma \mid X_{3}\right)\right]\left[1-F_{\gamma_{2, l}}(\gamma)\right]
$$

With the assumption of independent and identically distributed (i.i.d.) fading channels of the first hop from the secondary transmitter to any secondary relay, we also obtain $F_{\gamma_{1, l}}\left(\gamma \mid X_{3}\right)$ from (2) and (9) as

$$
F_{\gamma_{1, l}}\left(\left.\gamma\right|_{X_{3}}\right)=1-\exp \left(-\alpha_{1} \gamma x_{3} / \beta\right) \sum_{t=0}^{m_{1}-1} \frac{\alpha_{1}^{t}}{t !} \frac{\gamma^{t} x_{3}^{t}}{\beta^{t}}
$$

where $m_{1}$ and $\Omega_{1}$ are the fading severity and channel mean power of the link from $\mathrm{SU}_{\mathrm{TX}}$ to $\mathrm{SU}_{\mathrm{R}, l}$, respectively. Further, $\alpha_{1}$ is defined as $\alpha_{1}=m_{1} / \Omega_{1}$.

Now, we need to calculate the CDF of $\gamma_{2, l}$. As can be seen from (5), $\gamma_{2, l}$ is a function of random variables $\gamma_{2, l k}$, $k \in\{1, \ldots, K\}$. In addition, all $\gamma_{2, l k}$ with $k \in\{1, \ldots, K\}$ expressed in (4) have the same variable $X_{4, l}$, such that they are mutually dependent. Thus, we first calculate the CDF of $\gamma_{2, l}$ conditioned on $X_{4, l}$. Then, we obtain $F_{\gamma_{2, l}}(\gamma)$ as the expectation of $F_{\gamma_{2, l}}\left(\gamma \mid X_{4, l}\right)$ over the distribution of $X_{4, l}$. From (5), we have

$$
F_{\gamma_{2, l}}\left(\gamma \mid X_{4, l}\right)=\prod_{k=1}^{K} F_{\gamma_{2, l k}}\left(\gamma \mid X_{4, l}\right)=\left[F_{\gamma_{2, l k}}\left(\gamma \mid X_{4, l}\right)\right]^{K}
$$

Assume that the fading channels from $\mathrm{SU}_{\mathrm{R}, l}$ to any secondary receiver are i.i.d. with fading severity $m_{2, l}$ and channel mean power $\Omega_{2, l}$. From (4) and (9), $F_{\gamma_{2, l k}}\left(\gamma \mid X_{4, l}\right)$ is given by

$$
F_{\gamma_{2, l k}}\left(\left.\gamma\right|_{X_{4, l}}\right)=1-\exp \left(-\frac{\alpha_{2, l} \gamma x_{4, l}}{\beta}\right) \sum_{i=0}^{m_{2, l}-1} \frac{\alpha_{2, l}^{i}}{i !} \frac{\gamma^{i} x_{4, l}^{i}}{\beta^{i}}
$$

where $\alpha_{2, l}=m_{2, l} / \Omega_{2, l}$. Substituting (15) into (14), we have

$$
F_{\gamma_{2, l k}}\left(\left.\gamma\right|_{X_{4, l}}\right)=\left[1-\exp \left(-\frac{\alpha_{2, l} \gamma \mathrm{x}_{4, l}}{\beta}\right) \sum_{i=0}^{m_{2, l}-1} \frac{\alpha_{2, l}^{i}}{i !} \frac{\gamma^{i} x_{4, l}^{i}}{\beta^{i}}\right]^{K}
$$

By using the binomial expansion in [12, Eq. (1.111)], we can rewrite $F_{\gamma_{2, l k}}\left(\gamma \mid X_{4, l}\right)$ as

$$
\begin{aligned}
& F_{\gamma_{2, l k}}\left(\gamma \mid X_{4, l}\right)=1+\sum_{j=1}^{K} C_{j}^{K}(-1)^{j} \exp \left(-j \alpha_{2, l} \gamma x_{4, l} / \beta\right) \\
& \times\left[\sum_{i=0}^{m_{2, l}-1} \frac{\alpha_{2, l}^{i}}{i !} \frac{\gamma^{i} x_{4, l}^{i}}{\beta^{i}}\right]^{j}
\end{aligned}
$$

Using the identity product, we obtain $F_{\gamma_{2, l k}}\left(\gamma \mid X_{4, l}\right)$ as

$$
\begin{aligned}
& F_{\gamma_{2, l k}}\left(\left.\gamma\right|_{X_{4, l}}\right)=1+\sum_{j=1}^{K} C_{j}^{K}(-1)^{j} \exp \left(-j \alpha_{2, l} \gamma x_{4, l} / \beta\right) \\
& \times \underbrace{\sum_{i_{1}=0}^{m_{2, l}-1} \cdots \sum_{i_{j}=0}^{m_{2, l}-1}}_{j} \frac{1}{\prod_{w=1}^{j} i_{w} !} \frac{\gamma^{\sum_{w=1}^{j} i_{w}} \alpha_{2, l}^{\sum_{w=1}^{j} i_{w}} x_{4, l}^{\sum_{w=1}^{j} i_{w}}}{\beta^{\sum_{w=1}^{j} i_{w}}}
\end{aligned}
$$

Therefore, $F_{\gamma_{2, l}}(\gamma)$ is obtained as

$$
F_{\gamma_{2, l k}}(\gamma)=\int_{0}^{\infty} F_{\gamma_{2, l k}}\left(\left.\gamma\right|_{X_{4, l}}\right) f_{X_{4, l}}\left(x_{4, l}\right) d x_{4, l}
$$

Substituting (10) and (18) into (19), and then, applying [12, Eq. (3.381.4)] to calculate the resulting integral, we finally obtain $F_{\gamma_{2, l k}}(\gamma)$ as

$$
\begin{gathered}
F_{\gamma_{2}, l k}(\gamma)=1+\sum_{j=1}^{K} C_{j}^{K}(-1)^{j} \underbrace{\sum_{i_{1}=0}^{m_{2, l}-1} \cdots \sum_{i_{j}=0}^{m_{2, l}-1} \frac{\beta^{m_{4, l}} \alpha_{4, l}^{m_{4, l}}}{\prod_{w=1}^{j} i_{w} ! \alpha_{2, l}^{m_{4, l}}}}_{j} \\
\times \frac{\Gamma\left(m_{4, l}+\sum_{w=1}^{j} i_{w}\right)}{\gamma^{m_{4, l}+\sum_{w=1}^{j} i_{w=1} i_{w}} \Gamma\left(m_{4, l}\right)} \frac{\gamma^{i_{w}}}{\left(\gamma+\alpha_{4, l} \beta /\left(j \alpha_{2, l}\right)\right)^{m_{4, l}+\sum_{w=1}^{j} i_{w}}}
\end{gathered}
$$

where $m_{4, l}$ and $\Omega_{4, l}$ are, respectively, fading severity and channel mean power of the link from $\mathrm{SU}_{\mathrm{R}, l}$ to the primary receiver and $\alpha_{4, l}=m_{4, l} / \Omega_{4, l}$. By substituting (20) and (13) into (12), we obtain $F_{\gamma_{l}}\left(\left.\gamma\right|_{X_{3}}\right)$ as

$$
\begin{aligned}
& F_{\gamma_{l}}\left(\left.\gamma\right|_{X_{3}}\right)=1+\exp \left(-\frac{\alpha_{1} \gamma x_{3}}{\beta}\right) \sum_{t=0}^{m_{1}-1} \frac{\alpha_{1}^{t}}{t !} \frac{\gamma^{t} x_{3}^{t}}{\beta^{t}} \sum_{j=1}^{K} C_{j}^{K} \\
& \times(-1)^{j} \underbrace{\sum_{i_{1}=0}^{m_{2, l}-1} \cdots \sum_{i_{j}=0}^{m_{2, l}-1} \frac{1}{\prod_{w=1}^{j} i_{w} ! j^{m_{4, l}+\sum_{w=1}^{j} i_{w}} \alpha_{2, l}^{m_{4, l}}}}_{j} \\
& \times \frac{\Gamma\left(m_{4, l}+\sum_{w=1}^{j} i_{w}\right) \gamma^{\sum_{w=1}^{j} i_{w}}}{\Gamma\left(m_{4, l}\right)\left(\gamma+\alpha_{4, l} \beta /\left(j \alpha_{2, l}\right)\right)^{m_{4, l}+\sum_{w=1}^{j} i_{w}}}
\end{aligned}
$$

Substituting (21) into (11) and then applying the identity product, we obtain $F_{\gamma_{D}}\left(\gamma \mid X_{3}\right)$ as in (22). Therefore, $F_{\gamma_{D}}(\gamma)$ can be calculated as

$$
F_{\gamma_{D}}(\gamma)=\int_{0}^{\infty} F_{\gamma_{D}}\left(\left.\gamma\right|_{X_{3}}\right) f_{X_{3}}\left(x_{3}\right) d x_{3}
$$

Finally, substituting (22) and (10) into (23) together with the help of [12, Eq. (3.38.4)], we obtain an expression of $F_{\gamma_{D}}(\gamma)$ as in (24), where $m_{3}$ and $\Omega_{3}$ are, respectively, fading severity and channel mean power of the link from the secondary transmitter to the primary receiver and $\alpha_{3}=m_{3} / \Omega_{3}$.

\section{A. Outage Probability Performance}

Outage probability is defined as the probability that the instantaneous SNR falls below a predefined threshold $\gamma_{t h}$. Therefore, outage probability of the system can be obtained by using $\gamma_{t h}$ as the argument of $F_{\gamma_{D}}(\gamma)$ in (24) as $F_{\gamma_{D}}\left(\gamma_{t h}\right)$.

\section{B. Symbol Error Rate}

As reported in [14], SER can be directly expressed in terms of the CDF of the instantaneous SNR $\gamma_{D}$ as follows:

$$
P_{e}=\frac{a \sqrt{b}}{2 \sqrt{\pi}} \int_{0}^{\infty} F_{\gamma_{D}}(\gamma) \gamma^{-\frac{1}{2}} \exp (-b \gamma) d \gamma
$$

where $a$ and $b$ are modulation parameters, i.e., for $M$-PSK, $a=2, b=\sin ^{2}(\pi / M)$. In order to compute the SER, we 


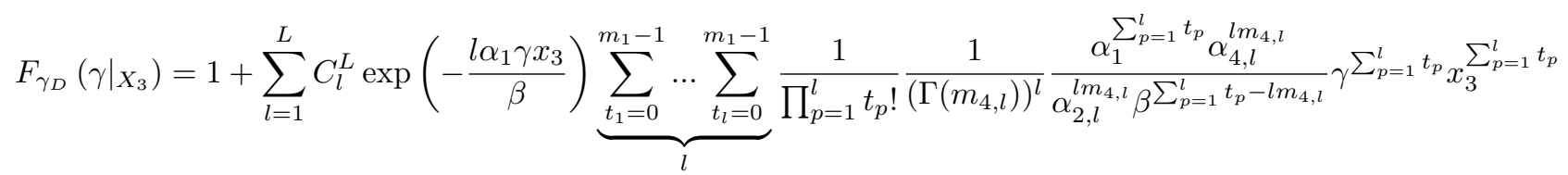

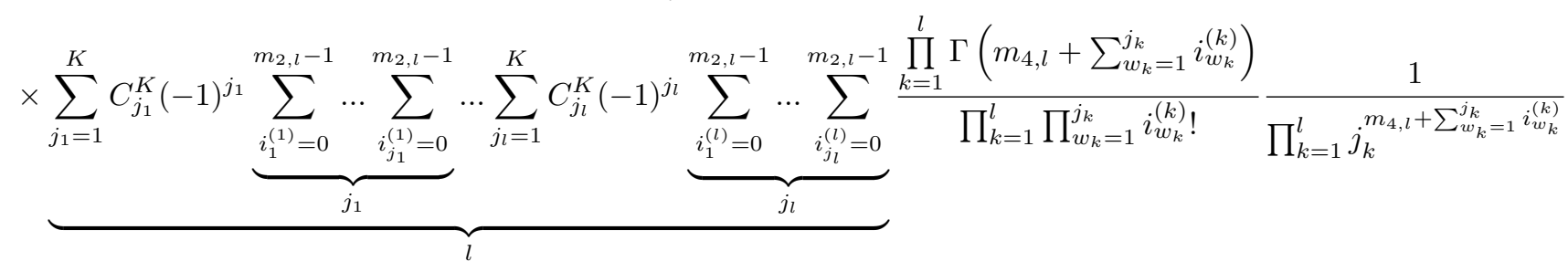

$$
\begin{aligned}
& \times \frac{\gamma^{\sum_{k=1}^{l} \sum_{w_{k}=1}^{j_{k}} i_{w_{k}}^{(k)}}}{\prod_{k=1}^{l}\left(\gamma+\frac{\alpha_{4, l} \beta}{j_{k} \alpha_{2, l}}\right)^{m_{4, l}+\sum_{w_{k}=1}^{j_{k}} i_{w_{k}}^{(k)}}}
\end{aligned}
$$

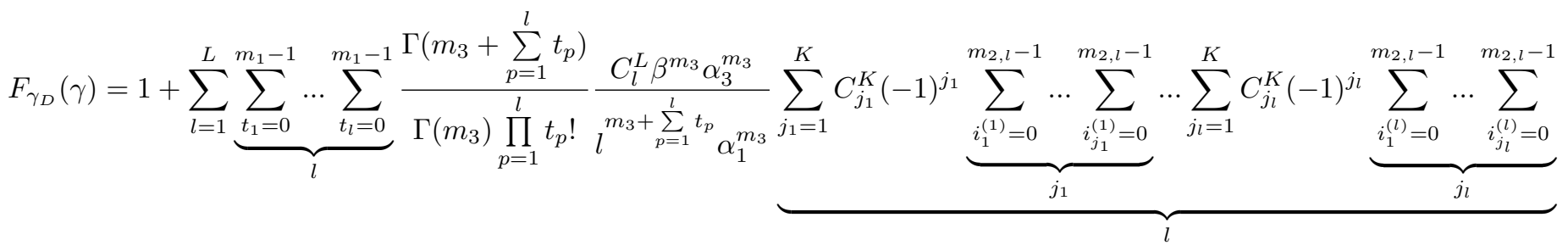

$$
\begin{aligned}
& \times \frac{\beta^{l m_{4, l}} \alpha_{4, l}^{l m_{4, l}} \prod_{k=1}^{l} \Gamma\left(m_{4, l}+\sum_{w_{k}=1}^{j_{k}} i_{w_{k}}^{(k)}\right)}{\alpha_{2, l}^{l m_{4, l}}\left(\Gamma\left(m_{4, l}\right)\right)^{l} \prod_{k=1}^{l} \prod_{w_{k}=1}^{j_{k}} i_{w_{k}}^{(k)} !} \frac{1}{\prod_{k=1}^{l} j_{k}^{m_{4, l}+\sum_{w_{k}=1}^{j_{k}} i_{w_{k}}^{(k)}}} \frac{\gamma^{\sum_{k=1}^{l} \sum_{w_{k}=1}^{j_{k}} i_{w_{k}}^{(k)}+\sum_{p=1}^{l} t_{p}}}{\left(\gamma+\frac{\beta \alpha_{3}}{l \alpha_{1}}\right)^{m_{3}+\sum_{p=1}^{l} t_{p}} \prod_{k=1}^{l}\left(\gamma+\frac{\alpha_{4, l} \beta}{j_{k} \alpha_{2, l}}\right)^{m_{4, l}+\sum_{w_{k}=1}^{j_{k}} i_{w_{k}}^{(k)}}}
\end{aligned}
$$

first substitute the CDF of the instantaneous SNR $\gamma_{D}$ in (24) into (25) along with applying [12, eq. (3.381.4)] to calculate the first integral, after some algebraic modifications, we can rewrite (25) as

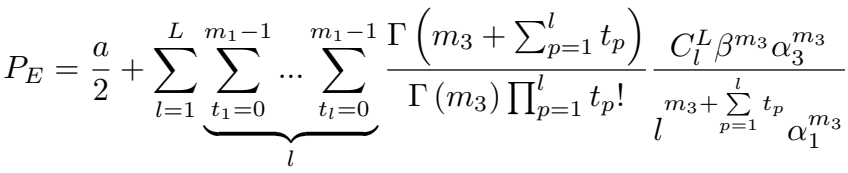

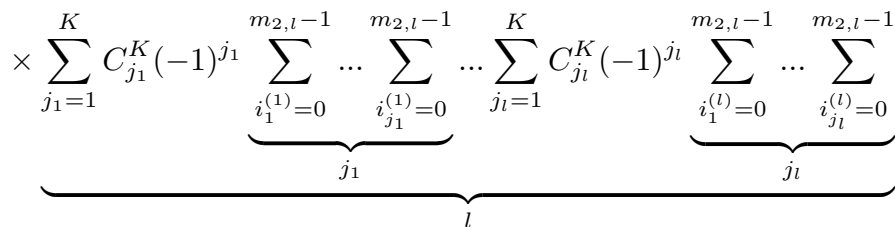

$$
\begin{aligned}
& \times \frac{\prod_{k=1}^{l} \Gamma\left(m_{4, l}+\sum_{w_{k}=1}^{j_{k}} i_{w_{k}}^{(k)}\right)}{\Gamma^{l}\left(m_{4, l}\right) \prod_{k=1}^{l} \prod_{w_{k}=1}^{j_{k}} i_{w_{k}}^{(k)} !} \frac{\beta^{l m_{4, l}} \alpha_{4, l}^{l m_{4, l}}}{\prod_{k=1}^{l} j_{k}^{m_{4, l}+\sum_{w_{k}=1}^{j_{k}} i_{w_{k}}^{(k)}} \alpha_{2, l}^{l m_{4, l}}} \\
& \times \int_{0}^{\infty} \frac{\gamma^{\sum_{k=1}^{l} \sum_{w_{k}=1}^{j_{k}} i_{w_{k}}^{(k)}+\sum_{p=1}^{l} t_{p}-\frac{1}{2}} \exp (-b \gamma)}{\left(\gamma+\frac{\beta \alpha_{3}}{l \alpha_{1}}\right)^{m_{3}+\sum_{p=1}^{l} t_{p}} \prod_{k=1}^{l}\left(\gamma+\frac{\alpha_{4, l} \beta}{j_{k} \alpha_{2, l}}\right)^{m_{4, l}+\sum_{w_{k}=1}^{j_{k}} i_{w_{k}}^{(k)}}} d \gamma
\end{aligned}
$$

Utilizing [12, Eq. (2.102)] to transform the integral expression of (26) into tabulated forms, then, we apply [15, Eq. (2.3.6.9)]

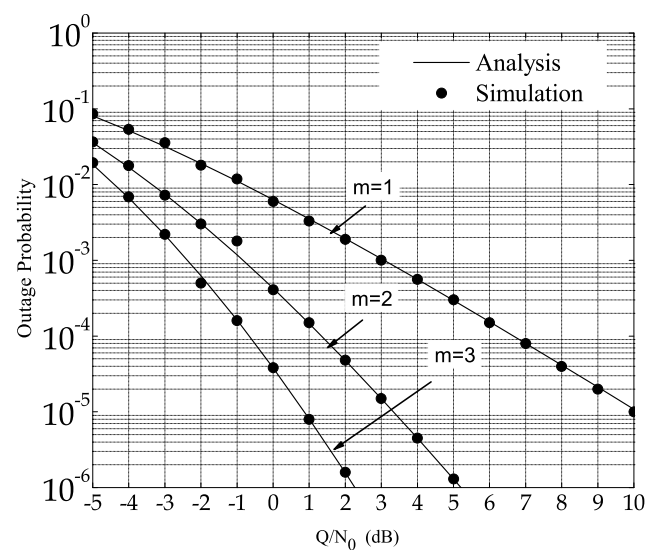

Fig. 2. Outage probability of the cognitive multiple relay system versus $Q / N_{0}$ for various fading severity parameters $m$.

to calculate the resulting integrals which leads to an expression for the SER of the system as in (27). The partial fraction coefficients $\chi_{r}$ and $\kappa_{q_{k}}^{(k)}$ in (27) are defined as (28).

\section{Numerical Results}

In this section, we provide numerical results to illustrate the system performance for various scenarios. The SNR threshold 


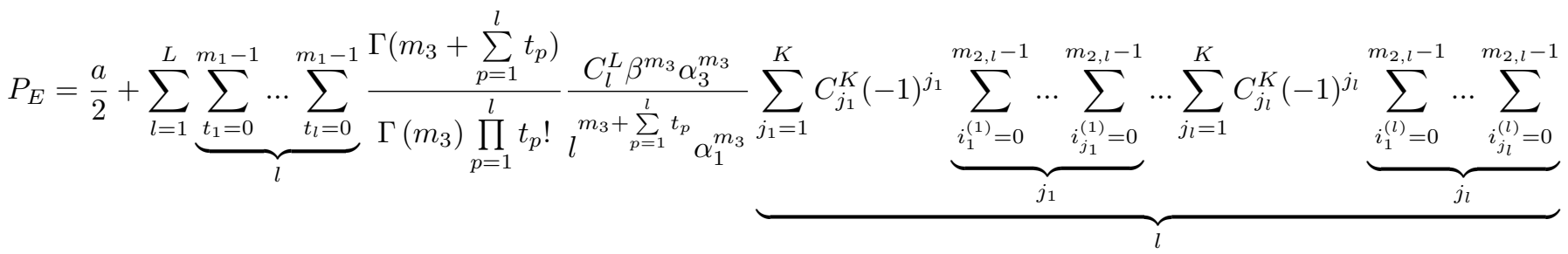

$$
\begin{aligned}
& \times \frac{\prod_{k=1}^{l} \Gamma\left(m_{4, l}+\sum_{w_{k}=1}^{j_{k}} i_{w_{k}}^{(k)}\right)}{\prod_{k=1}^{l} \prod_{w_{k}=1}^{j_{k}} i_{w_{k}}^{(k)} !} \frac{\beta^{l m_{4, l}} \alpha_{4, l}^{l m_{4, l}}}{\Gamma^{l}\left(m_{4, l}\right) \prod_{k=1}^{l} j_{k}^{m_{4, l}+\sum_{w_{k}=1}^{j_{k}} i_{w_{k}}^{(k)}} \alpha_{2, l}^{k m_{4, l}}}\left\{\sum_{r=1}^{m_{3}+\sum_{p=1}^{l} t_{p}} \chi_{r} \Gamma\left(\sum_{k=1}^{l} \sum_{w_{k}=1}^{j_{k}} i_{w_{k}}^{(k)}+\sum_{p=1}^{l} t_{p}+\frac{1}{2}\right)\right. \\
& \times\left(\frac{\beta \alpha_{3}}{l \alpha_{1}}\right)^{\sum_{k=1}^{l} \sum_{w_{k}=1}^{j_{k}} i_{w_{k}}^{(k)}+\sum_{p=1}^{l} t_{p}+\frac{1}{2}-r} U\left(\sum_{k=1}^{l} \sum_{w_{k}=1}^{j_{k}} i_{w_{k}}^{(k)}+\sum_{p=1}^{l} t_{p}+\frac{1}{2}, \sum_{k=1}^{l} \sum_{w_{k}=1}^{j_{k}} i_{w_{k}}^{(k)}+\sum_{p=1}^{l} t_{p}+\frac{3}{2}-r, b \frac{\beta \alpha_{3}}{l \alpha_{1}}\right)+\sum_{k=1}^{l} \sum_{q_{k}=1}^{m_{4, l}+\sum_{w_{k}=1}^{j_{k}} i_{w_{k}}^{(k)}} \\
& \times \kappa_{q_{k}}^{(k)} \Gamma\left(\sum_{k=1}^{l} \sum_{w_{k}=1}^{j_{k}} i_{w_{k}}^{(k)}+\sum_{p=1}^{l} t_{p}+\frac{1}{2}\right)\left(\frac{\alpha_{4, l} \beta}{j_{k} \alpha_{2, l}}\right)^{\sum_{k=1}^{l} \sum_{w_{k}=1}^{j_{k}} i_{w_{k}}^{(k)}+\sum_{p=1}^{l} t_{p}+\frac{1}{2}-q_{k}} U\left(\sum_{k=1}^{l} \sum_{w_{k}=1}^{j_{k}} i_{w_{k}}^{(k)}+\sum_{p=1}^{l} t_{p}+\frac{1}{2}, \sum_{k=1}^{l} \sum_{w_{k}=1}^{j_{k}} i_{w_{k}}^{(k)}\right. \\
& \left.\left.+\sum_{p=1}^{l} t_{p}+\frac{3}{2}-q_{k}, b \frac{\alpha_{4, l} \beta}{j_{k} \alpha_{2, l}}\right)\right\}
\end{aligned}
$$

$$
\begin{gathered}
\chi_{r}=\frac{1}{\left(m_{3}+\sum_{p=1}^{l} t_{p}-r\right) !} \frac{d^{m_{3}+\sum_{p=1}^{l} t_{p}-r}}{d \gamma^{m_{3}+\sum_{p=1}^{l} t_{p}-r}}\left[\frac{1}{\prod_{k=1}^{l}\left(\gamma+\frac{\alpha_{4, l} \beta}{j_{k} \alpha_{2, l}}\right)^{m_{4, l}+\sum_{w_{k}=1}^{j_{k}} i_{w_{k}}^{(k)}}}\right]_{\gamma=-\frac{\alpha_{4, l} \beta}{j_{k} \alpha_{2, l}}} \\
\kappa_{q_{k}}^{(k)}=\frac{1}{\left(m_{4, l}+\sum_{w_{k}=1}^{j_{k}} i_{w_{k}}^{(k)}-q_{k}\right) !} \frac{d^{m_{4, l}+\sum_{w_{k}=1}^{j_{k}} i_{w_{k}}^{(k)}-q_{k}}}{d \gamma^{m_{4, l}+\sum_{w_{k}=1}^{j_{k}} i_{w_{k}}^{(k)}-q_{k}}}\left[\frac{\left(\gamma+\frac{\alpha_{4, l} \beta}{j_{k} \alpha_{2, l}}\right)^{m_{4, l}+\sum_{w_{k}=1}^{j_{k}} i_{w_{k}}^{(k)}}}{\left.\left(\gamma+\frac{\beta \alpha_{3}}{l \alpha_{1}}\right)^{m_{3}+\sum_{p=1}^{l} t_{p}} \prod_{k=1}^{l}\left(\gamma+\frac{\alpha_{4, l} \beta}{j_{k} \alpha_{2, l}}\right)^{m_{4, l}+\sum_{w_{k}=1}^{j_{k}} i_{w_{k}}^{(k)}}\right]_{\gamma=-\frac{\alpha_{4, l} \beta}{j_{k} \alpha_{2, l}}}}\right.
\end{gathered}
$$

for calculating the outage probability is fixed as $\gamma_{t h}=3$ $\mathrm{dB}$ for all the examples. Let the normalized distances of the links $\mathrm{SU}_{\mathrm{TX}} \rightarrow \mathrm{SU}_{\mathrm{R}, l}, \mathrm{SU}_{\mathrm{R}, l} \rightarrow \mathrm{SU}_{\mathrm{RX}, k}, \mathrm{SU}_{\mathrm{TX}} \rightarrow \mathrm{PU}_{\mathrm{RX}}$, and $\mathrm{SU}_{\mathrm{R}, l} \rightarrow \mathrm{PU}_{\mathrm{RX}}$ be denoted as $d_{1}, d_{2}, d_{3}$, and $d_{4}$, respectively. We assume that the summation of the transmission distances from secondary transmitter to relay and from the relay to receiver is normalized to unity, i.e., $d_{1}+d_{2}=1$. Assume that the channel mean powers are mainly effected by path loss and shadowing, i.e., all channel mean powers are attenuated according to the exponential decaying model with path loss exponent of $n$, i.e., $\Omega_{l}=d_{l}^{-n}$. Based on empirical measurements in [16], depending on the outdoor propagation environment, $n$ can assume a value from the set $\{1,2,3,4\}$. In our numerical examples, we select $n=4$ for highly shadowed urban environment and the transmission distances are chosen as $d_{1}=d_{2}=0.5$ and $d_{3}=d_{4}=1.0$.

Fig. 2 and Fig. 3 plot outage probability and SER versus interference power-to-noise ratio $Q / N_{0}$. The examined net- work has 3 relays, $L=3$, and 5 secondary receivers, $K=5$. The fading severity parameters are selected to be the same as $m_{1}=m_{2}=m_{3}=m_{4}=m$, i.e., $m=1$ for the scenario that the system operates in a densely populated area with the most severe multi-path effect. For the case that the system operates in a moderate environment with less scattering and multi-path effect, the fading severity parameter is set as $m=2$. Finally, $m=3$ is selected for the situation that the system operates in a dominant line-of-sight environment. As expected, the best performance is obtained for the case of $m=3$ and the outage probability and SER are highest in the case of $m=1$.

Fig. 4 and Fig. 5, respectively, examine the impact of the number of secondary relays and secondary receivers on outage probability and SER. To make a fair comparison, fading severity parameters are fixed as $m_{1}=m_{2}=m_{3}=m_{4}=2$ for all examined examples. In these figures, as the interference power-to-noise ratio $Q / N_{0}$ increases, the outage probability and SER performance are improved. Furthermore, it can be 


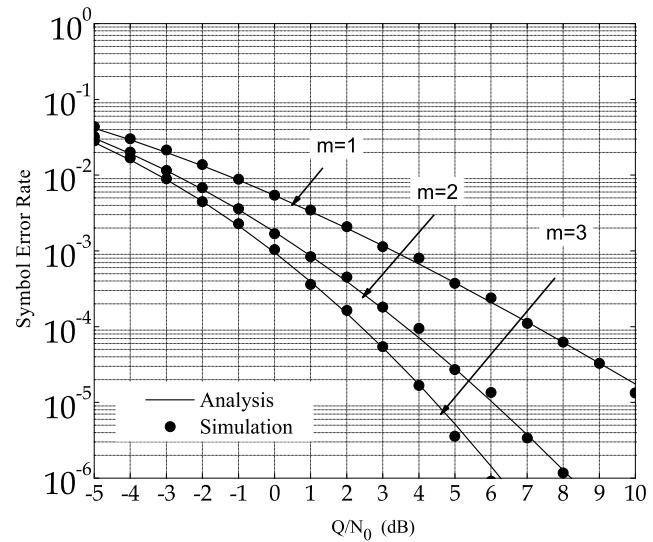

Fig. 3. Symbol error rate of $Q P S K$ modulation of the cognitive multiple relay system versus $Q / N_{0}$ for various fading severity parameters $m$.

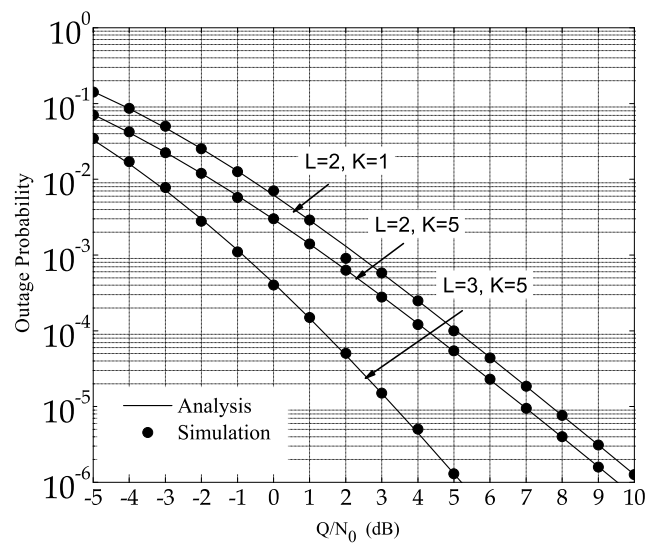

Fig. 4. Outage probability of the cognitive multiple relay system versus $Q / N_{0}$ for various number of relays, $L$, and secondary receivers, $K$.

observed from Fig. 4 and Fig. 5 that outage probability decreases as the number of secondary relays and receivers increases. This is due to the fact that with a larger number of secondary relays and receivers also the number of relaying routes increases. This, in turn, results in a potentially higher end-to-end SNR at the selected secondary destination.

\section{CONCLUSIONS}

In this paper, we have conducted a performance analysis for a CCRN with multi-receiver scheduling. Specifically, only one relay and one secondary receiver which offer the highest instantaneous signal-to-noise ratio is scheduled to transmit signals at a particular time instant. We deploy multiple DF relays to assist the secondary transmitter in communicating with multiple secondary receivers. Furthermore, we have investigated system performance by deriving expressions for outage probability and SER over Nakagami- $m$ fading. Numerical results are also provided to illustrate the effects of fading conditions, the number of relays and secondary receivers on the secondary system performance.

\section{REFERENCES}

[1] A. Martian, I. Marcu, and I. Marghescu, "Spectrum occupancy in an urban environment: A cognitive radio approach," in Advanced Interna-

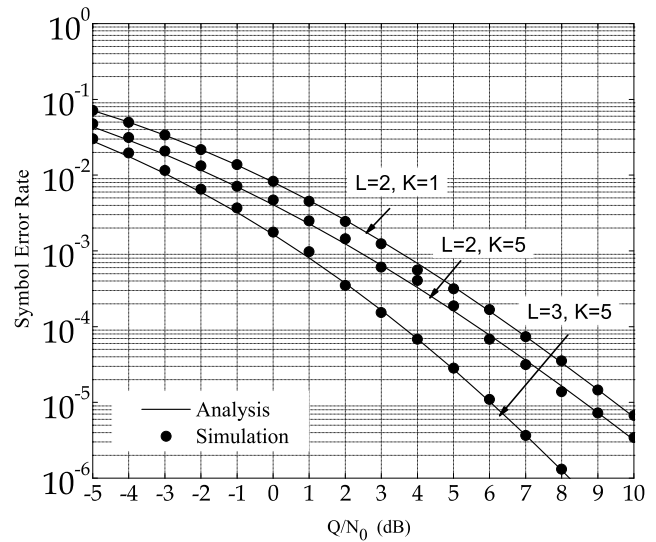

Fig. 5. Symbol error rate of $Q P S K$ modulation of the cognitive multiple relay system versus $Q / N_{0}$ for various number of relays, $L$, and secondary receivers, $K$.

tional Conference on Telecommunications, Barcelona, Spain, May 2010, pp. 25-29.

[2] J. Mitola, "Cognitive radio for flexible mobile multimedia communications," in Proc. IEEE International Workshop on Mobile Multimedia Communications, San Diego, USA, Nov. 1999, pp. 3-10.

[3] S. Haykin, "Cognitive radio: Brain-empowered wireless communications," IEEE J. Sel. Areas Commun., vol. 23, no. 2, pp. 201-220, Feb. 2005.

[4] T. M. C. Chu, H. Phan, and H.-J. Zepernick, "On the performance of underlay cognitive radio networks using M/G/1/K queueing model," IEEE Communications Letters, vol. 17, no. 5, pp. 876-879, May 2013.

[5] R. Zhang, "On peak versus average interference power constraints for protecting primary users in cognitive radio networks," IEEE Trans. Wireless Commun., vol. 8, no. 4, pp. 2112-2120, Apr. 2009.

[6] T. M. C. Chu, H. Phan, and H.-J. Zepernick, "Opportunistic spectrum access for cognitive amplify-and-forward relay networks," in Proc. IEEE Vehicular Technology Conference, Dresden, Germany, Jun. 2013, pp. 15 .

[7] L. Li, F. A. Khan, M. Pesavento, and T. Ratnarajah, "Power allocation and beamforming in overlay cognitive radio systems," in Proc. IEEE Vehicular Technology Conference, Budapest, Hungary, May 2011, pp. $1-5$.

[8] T. M. C. Chu, H. Phan, and H.-J. Zepernick, "Hybrid interweaveunderlay spectrum access for cognitive cooperative radio networks," IEEE Trans. Commun., vol. PP, no. 99, May 2014.

[9] J. N. Laneman, D. N. C. Tse, and G. W. Wornell, "Cooperative diversity in wireless networks: Efficient protocols and outage behavior," IEEE Trans. Inf. Theory, vol. 50, no. 12, pp. 3062-3080, Dec. 2004.

[10] J. Lee, H. Wang, J. G. Andrews, and D. Hong, "Outage probability of cognitive relay networks with interference constraints," IEEE Trans. Wireless Commun., vol. 10, no. 2, pp. 390-395, Feb. 2011.

[11] S. Sagong, J. Lee, and D. Hong, "Capacity of reactive DF scheme in cognitive relay networks," IEEE Trans. Wireless Commun., vol. 10, no. 10 , pp. 3133-3138, Oct. 2011.

[12] I. S. Gradshteyn and I. M. Ryzhik, Table of Integrals, Series, and Products, 7th ed. Academic Press, 2007.

[13] T. Wang, A. Cano, G. B. Giannakis, and J. N. Laneman, "Highperformance cooperative demodulation with decode-and-forward relays," IEEE Trans. Commun., vol. 55, no. 7, pp. 1427-1438, Jul. 2007.

[14] R. H. Y. Louie, Y. Li, H. A. Suraweera, and B. Vucetic, "Performance analysis of beamforming in two hop amplify and forward relay networks with antenna correlation," IEEE Trans. Wireless Commun., vol. 8, no. 6, pp. 3132-3141, Jun. 2009.

[15] A. P. Prudnikov, Y. A. Brychkov, and O. I. Marichev, Integrals and Series, vol. 1. New York: Gordon and Breach Science Publishers, 1986.

[16] X. Zhao, J. Kivinen, P. Vainikainen, and K. Skog, "Propagation characteristics for wideband outdoor mobile communications at $5.3 \mathrm{GHz}$," IEEE J. Sel. Areas Commun., vol. 20, no. 3, pp. 507-514, Apr. 2002. 